\title{
Brian Connaughton (coord.), Repensando Guatemala en la época de Rafael Carrera. El país, el hombre y las coordenadas de su tiempo, México, UAM/Gedisa, 2015, 524 pp.
}

No es frecuente encontrar un libro sobre la historia de Guatemala en el siglo XIX publicado desde México y con los alcances que tiene el que tenemos entre manos. Para corroborar esto, basta con revisar la bibliografía general que se ofrece al final del volumen, que da cuenta de un buen número de libros publicados sobre todo en Guatemala, amen de los anotados a pie de página que hablan de la enorme producción norteamericana sobre el tema. En ese sentido, realmente estamos ante una obra que resulta por demás importante para la historiografía decimonónica, pues los autores, en esta obra coordinada por Brian Connaughton, desde diferentes ángulos y perspectivas, nos ofrecen miradas renovadas y esclarecedoras sobre uno de los periodos más trascendentales de la historia guatemalteca, el que transcurre entre 1831 y 1871: llamado “de la época de Rafael Carrera”. Lapso también caracterizado como de conservadurismo, lo que no significaba necesariamente continuidad, sino como bien se señala en la introducción, fue una de profundos desequilibrios, convulsiones, cambios y "transformaciones étnicas". Yo diría, de profundas tensiones estructurales entre lo preexistente y lo que se quería imponer como nuevo.

Producto mayormente de un Coloquio celebrado en 2013, los autores de Repensando Guatemala en la época de Rafael Carrera..., sostienen un diálogo permanente con Ralph Lee Woodward Jr., destacado historiador norteamericano quien hace dos décadas escribió una de las obras más relevantes que existen sobre esa historia. Reconociendo esa deuda, los autores del libro que aquí reseñamos realmente buscan "Repensar" diversos aspectos de dicho periodo, algunos que aún quedaban pendientes, o bien otros a los que había que volver con nuevas preguntas o la relectura de fuentes para profundizar en ellos, pues de la obra de Woodward a esta median al menos veinte años.

Dividido en cinco apartados, la Introducción y una Coda, el volumen en su conjunto aborda el análisis de la descomposición del orden social colonial en Guatemala desde 1831 y hasta 1871. Periodo en el que lo mismo hubo un levantamiento popular en las montañas, "zozobra por la fragilidad de la república en Centroamérica” y finalmente, como sabemos, la 
disolución de ésta en pequeños estados que hasta la fecha nunca se han consolidado del todo.

Importa decir que la Introducción y la Coda, de la autoría ambas de Brian Connauhgton, ofrecen, la primera, una inteligente síntesis de todos los trabajos que integran el volumen, mientras que la Coda al final los sitúa en el conjunto de la vasta historiografía que existe no solo sobre Guatemala y Centroamérica, sino en comparación con la de las mismas temáticas para el caso mexicano. De tal suerte que ofrecen como debe ser, no solo el panorama completo de todo el conjunto sino los alcances y las perspectivas que dejan abiertas para futuras investigaciones. Sin embargo, nuestra tarea consiste en reseñar el contenido, lo que enseguida hacemos.

En el primer apartado, Adolfo Bonilla en su texto titulado "Triunfos y fracasos de la política ilustrada centroamericana (1774-1838)", aborda el quiebre de la república federal a partir del análisis de dos fases de la ilustración: la primera la fase brillante del despotismo ilustrado que fue imponiendo ciertas condiciones materiales e ideológicas para que se fomentaran las ideas liberales y republicanas; la segunda, a partir de 1829, llevó al triunfo del absolutismo ilustrado y a la derrota del liberalismo. Vino entonces una etapa de imposición de reformas "mal concebidas", preparadas y organizadas que afectaron los intereses de la población indígena, ladina y mulata de oriente, que llevó al levantamiento liderado por Teodoro Mejía y Rafael Carrera a partir de 1836. La historia de este proceso, que llevó al quiebre de la unidad centroamericana y a la desaparición de las condiciones de la república federal, es analizado con detalle por Bonilla.

El segundo apartado titulado "Nuevos actores", consta de dos colaboraciones: en la primera, Ann Jefferson, en un sensible ensayo titulado "Nuestra América: la visión de la gente parda del Distrito de Mita, 1837”, que une la preocupación por el presente, se propone analizar una tercera visión sobre la tierra, no la de los liberales o conservadores, sino aquella que tenían aquellos actores más cercanos a ella, que todavía no la veían como una mercancía sino como parte de un cosmos que estaba en peligro de desaparecer. Se trata sobre todo de encontrar el sentido que los pardos tenían sobre el concepto "Nuestra América”, que les llevó a defenderla de las políticas liberales mediante el levantamiento armado, con Tomás Mexía y luego Rafael Carrera como líderes de ellos y llevando de esa forma a detener el proyecto liberal al menos por 30 años. Lo que no significó "conservarse” en el pasado. 
En "Rafael Carrera, de "indio" a presidente vitalicio, la metamorfosis del hombre y la cultura política”, Brian Connaughton busca recuperar en fuentes de la época la evolución que tuvo la personalidad y actuación de este importante personaje que marcó todo ese periodo. Carrera, alrededor de cuya época gira todo el libro, fue central en la caída de la federación y el declive del liberalismo durante tres décadas. Resulta a todas luces un personaje polémico, cuya trayectoria no queda desligada de los procesos que vivían Guatemala y Centroamérica en el periodo. El análisis de Connaughton lleva a esclarecer los motivos de la aversión que importantes sectores de la élite sentían tanto hacia el régimen como ante quien lo encabezaba. No sólo era la violencia que marcó el momento sino el que estuviera al mando un representante de sectores sociales que hasta ese punto habían sido subordinados. Desde distintas visiones, el autor va encontrando el perfil que se le iba asignando a este personaje de origen "popular", en diferentes momentos. En palabras de un conservador criollo de la época, recogidas por Connaughton, se trataba de un individuo en transición, que se fue formando mediante la asimilación de elementos tanto de la política como de los militares que terminaron por configurar su particular cultura política. Nunca fue su trayectoria estática ni monolítica, por el contrario, resultaba acomodaticia. Las características del desarrollo de esa personalidad emergen con claridad tras el revelador análisis de las diversas fuentes, bien de criollos exilados o de viajeros extranjeros que realiza el autor.

Un tercer apartado aborda en tres ensayos, con maestría y versatilidad, los siempre difíciles asuntos de la fiscalidad y la participación militar. En "Santa Rosa y Chiquimula, participación fiscal y militar, 1839-1870”, Juan Carlos Sarazúa explora los asuntos fiscales y militares de esos dos departamentos porque, señala, la historiografía ha apuntado los importantes aportes que hicieron de soldados y recursos, pero el autor trata de hacerlo con más detalle y lo que puede considerarse un objetivo más de fondo, como es discutir con base en ello algunos puntos sobre el orden construido por Carrera y la élite conservadora -Iglesia y comerciantes- en la época. Estas alianzas, nos dice el autor, fueron el trasfondo que permitió encontrar cierto orden en el régimen vigente y son clave para entenderlo.

Así, mediante un análisis minucioso de los elementos propuestos -fiscalidad y participación militar-, Sarazúa nos muestra una historia en la que no solamente importa el poder estatal sino el local, sobre todo, apunta a que entendamos el mundo social local en sus complejas interacciones; la relación entre impuestos y servicio militar, así como el papel de la 
guerra visto desde una perspectiva social. Tales elementos le permiten al autor dejar abierto el camino para la comprensión de la etapa histórica subsecuente, la que inició en 1871 cuando los militares liberales tomaron el poder.

Por su parte, Moisés Ornelas en "Los asuntos fiscales en La Gaceta de Guatemala. De la guerra de la Montaña a la presidencia vitalicia. ¿Estabilidad económica igual a gobernabilidad? (1847-1858)", mediante una minuciosa revisión de los diferentes pero sistemáticos datos fiscales aportados por ese periódico oficial, busca ahondar en la situación fiscal y hacendaria que prevaleció durante esos años que permitan revalorar, más allá de la percepción meramente político-ideológica dominante en la historiografía sobre el régimen de Carrera, el verdadero estado que guardaba Guatemala en la época. Una detallada revisión y reconstrucción de los ingresos de la hacienda pública durante los años de Guerra -sobre todo centrados en la alcabala y el estanco de aguardiente, permiten a Ornelas identificar como se resolvió el gasto militar, punto central de esos momentos; en una segunda fase, el autor analiza con detalle la situación de la hacienda una vez pasada la guerra, momento en el que el naciente Estado necesitaba reorganizar y equilibrar la hacienda buscando la estabilidad, en medio de condiciones difíciles incluso de amenaza de invasiones extranjeras. La conclusión final del autor es que, efectivamente, echar luz sobre el tema fiscal, hacendario y económico, le permitió observar como una prioridad de los conservadores estuvo fincada en el proyecto económico y hacendario que, al final de cuentas, les permitió mantener la gobernabilidad, resultado distinto a lo alcanzado por los liberales.

Leticia González Sandoval en "Entre la moralidad y el interés fiscal. El estanco de aguardiente en el periodo conservador", revisa, en sus palabras, "las implicaciones que tuvo para la hacienda pública el tránsito del estanco de aguardiente del control estatal al privado y las relaciones sociales ligadas a su funcionamiento en ese periodo de formación del Estado.” De acuerdo con la autora, tres ejes cruzaban este asunto desde el siglo XVIII: los ingresos que generaba a la hacienda pública, el tema de la embriaguez, atribuida generalmente a los sectores bajos de la sociedad -es decir, indios y ladinos-, y el tema de la moralidad. Señala que en el tránsito hacia la situación independiente no cambió esto, sin embargo si lo hizo durante el periodo conservador, cuando la política al respecto privilegió al erario y dejó de lado la moral pública. Se trata de un abordaje sumamente interesante de un asunto que afectaba tanto a élites como a sectores populares de la población, pues representaba uno de los ingresos fiscales más notables de la hacienda pública y después de los particulares, 
quienes a la par construían una serie de mitos sobre la población proclive a ser consumidor. En estas transacciones que se hicieron entre autoridades, dueños de los estancos y pueblos, estos últimos también tuvieron oportunidad de tener en algunos momentos el control de ellos. Se examina incluso la venta clandestina de las bebidas embriagantes, así como la participación de cofradías y mujeres en su producción y distribución. Sin duda, se trata de un tema por demás complejo que atravesaba a toda la sociedad guatemalteca -y de cualquier otro lugar-, en la época.

Una vez revisados en el libro los temas hacendarios, fiscales y económicos, el siguiente apartado "Vida cotidiana ante guerras, gobiernos y políticas", se torna "más amable”, por decirlo así, pues nos lleva de la mano en dos capítulos a pensar en otros actores y escenarios: por una parte, Lizeth Jiménez Chacón, examina la presencia y participación de la mujer en la construcción del Estado y en los momentos de violencia que vivió el país, en esos tiempos de fuerte y constante conflicto bélico, con el fin de responder a una pregunta central ¿Cómo vivieron las mujeres esos tiempos? ¿Cambiaba su vida con ellos? A ellas las encuentra en diferentes escenarios, incluso aquellos considerados "contrarios a su naturaleza”, que, por otra parte, estaba siendo redefinida en esas décadas. La situación de bandolerismo, en la que sigue el modelo de Hobsbwam, fue otro fenómeno que trastocó también la vida de las mujeres donde cumplían al menos tres roles: el de amantes, colaboradoras y enlaces y, singularmente, bandoleras. Un aspecto más que es analizado en torno a las mujeres es su situación de viudez tan frecuente en una sociedad en guerra, como la que experimentaba Guatemala en la época de Carrera. La guerra, pues, concluye la autora, afectaba más a las mujeres en tanto el entorno estuviera más afectado por la violencia de diversos tipos que se vivía entonces.

El segundo ensayo, obra de Tania Sagastume Paiz, muestra los cambios y continuidades habidos en los esfuerzos de las autoridades por controlar y educar a la población mediante el análisis de la reglamentación sobre trabajo, vagancia y diversiones públicas a mediados del siglo XIX. Así, realiza una comparación con la etapa previa que va de fines del siglo XVIII al fin del primer gobierno liberal en 1838, etapa en la cual comenzaron a ser tocados estos aspectos. Las ideas y acciones que permearon esa legislación durante el gobierno conservador tenían que ver, señala la autora, con "el intento de desmantelar las reformas liberales, la recuperación que hizo la iglesia de su predominio en la educación y la sociedad, así como el restablecimiento de algunas leyes coloniales en materia 
de pueblos indígenas”. Enfoca el aspecto administrativo tanto de modificaciones en los mandos de gobierno en el nivel de corregidores, municipalidades así como los esfuerzos por controlar la vagancia. Ya en materia de las diversiones públicas y juegos prohibidos, también desde los reglamentos, revisa lo tocante a los teatros, los toros, las peleas de gallos y otras diversiones, destacando como se redujo la reglamentación sin que se eliminara el tono moralizador de la misma, aunque a medida que transcurrió el siglo se fue orientando hacia el orden, el cumplimiento de las leyes y la dedicación de todos al trabajo.

El último ensayo, a la vez último apartado, se titula "Los adoradores del sol": Santa Catarina Ixtahuacan durante el régimen conservador, de la autoría de Francisco Rodolfo González Galeotti. En este nos lleva a la región de "Los altos", donde se desarrolló una batalla de poder entre indígenas y gobernantes por la tierra y el cultivo del café. Sobretodo, analiza aspectos del proceso político social del pueblo mencionado en el título después de la independencia con la intención de comprender como la población maya del lugar “interactúo con agentes políticos-estatales y con otras poblaciones, en términos de alianzas y subalternidades.” Le interesa observar como esa población mutó en el siglo XIX creando alianzas o jerarquías nuevas en su interior acordes con situaciones externas e internas. Se trata sobre todo de la historia de los indígenas guatemaltecos vista desde un caso particular y quizá paradigmático.

En suma, el conjunto de textos reunidos en este libro cumple con los propósitos generales explicados en la introducción: comprender aquellas décadas centrales del siglo XIX guatemalteco con base en revisar lo antes hecho, y potencia un eje analítico dándole la centralidad que quizá no se le había dado, lo que se cumple con creces. Esto no quiere decir que no se necesita más investigación para ciertos temas, como, por ejemplo, el tema de la fiscalidad, expuesto desde la prensa en el texto de Moisés Ornelas, que necesitaría todavía profundizar desde otras fuentes; o el de vagos, malentretenidos y diversiones públicas en Guatemala, que a mi gusto, al ser analizado desde la legislación, pierde la perspectiva social y cultural que pudiera tener y que para alcanzarla también requiere de la consulta de otro tipo de fuentes, además de que podría hacerse comparativamente con los casos no sólo de la ciudad de México sino de Guadalajara y Zacatecas, para los que ya existen investigaciones.

No obstante, esto no resta ningún mérito al libro, que representa una importante e incisiva aportación no sólo a la historiografía sobre la historia de Guatemala en el siglo XIX, sino en general, a la de procesos de construcción de los Estados-Nación en América Latina. 
Enhorabuena a los autores por esta obra y a los interesados en la historia Centroamericana en particular.

Rosalina Ríos Zúñiga

Universidad Nacional Autónoma de México rorst5@hotmail.com 